\title{
Prediction of herb-drug interactions involving consumption of furanocoumarin-mixtures and cytochrome P450 1A2-mediated caffeine metabolism inhibition in humans
}

\section{Zeyad Ibrahim Alehaideb ( $\square$ zehaideb@gmail.com )}

KAIMRC https://orcid.org/0000-0002-7185-2820

\section{Research Article}

Keywords: Caffeine, Cytochrome 1A2 Inactivation, Furanocoumarin, Herb-Drug Interaction Prediction, Natural Products

Posted Date: August 23rd, 2021

DOI: https://doi.org/10.21203/rs.3.rs-836915/v1

License: (c) (1) This work is licensed under a Creative Commons Attribution 4.0 International License. Read Full License 


\section{Introduction}

Regulatory bodies require the investigation of drug-drug interactions (DDI) prior to new-drug-candidate approval (Health Canada, 1998; EMEA, 2010; USFDA, 2012). Based on regulatory guidelines, new-drug-applicants are required to follow a step-wise protocol which includes the investigation of major metabolizing enzymes inhibition, most notably cytochrome P450 (CYP) enzymes, in an effort to reduce unnecessary clinical trials and post-marketed drug-withdrawals. Several DDI prediction models have been developed by researchers and adopted by regulatory bodies. Until recently, DDI prediction is based mainly on reversible CYP enzyme inhibition mechanisms and has been carried out routinely as part of drug preclinical studies (Fahmi et al., 2008). If irreversible inhibition were to occur, this would result in underestimation of the magnitude of DDI (Einolf, 2007). Co-administration of a pharmaceutical drug and an herbal product, with bioactive constituents that interfere with drug metabolite(s) formation, might significantly alter the pharmacokinetics of the victim-drug. The outcome of such alterations may result in serious clinical consequence and deaths (Ebbesen et al., 2001).

Predictive DDI models have been developed with increased accuracy in prediction not only for reversible DDI but also for irreversible DDI. For example, Mayhew et al. (2000) reported a predictive DDI model which involves an irreversible inhibitor. Fahmi et al. (2008) proposed a combination model to sum up the inhibitory effects of reversible and irreversible enzyme inhibition along with enzyme induction. Despite these improvements, the DDI predictive model usually addresses interaction between one victim-drug and one inhibitor, which is not always the case in herb-drug interaction 
(HDI) as herbal products usually consist of a complex mixture of bioactive chemicals which may interact with the victim-drug.

To implement a predictive DDI model involving time-dependent inhibitors (TDI), or the mechanism-based inhibitors (MBI), requires information on in vitro inactivation kinetics of the victim-drug as well as in vivo hepatic inlet inhibitor concentration $\left([\mathrm{I}]_{\mathrm{H}}\right)$. In the case of an herbal extract, the challenge is to estimate the $[\mathrm{I}]_{\mathrm{H}}$ or an integrated dose of the bioactive chemicals of the herb in the liver. Wang et al. (2004) had modified the model of Mayhew et al. to account for a multitude of inhibitors, which probably can be applied to a mixture of bioactive inhibitors in an herbal extract. The DDI model of Wang et al. requires detailed pharmacokinetics information on each inhibitor in the herbal extract. These can be time-consuming and expensive at the early drug development stage. In this study, we propose to use the concentration-addition (CA) model (Safe, 1998; ATSDR, 2004; Alehaideb et al., 2019) to calculate an integrated dose/concentration for the furanocoumarin-mixture in the liver. The integrated dose is then used to calculate the area-under-curve-ratio (AUCR) with the Mayhew et al. model.

The "victim-drug" in this study is caffeine. A popular drug with adverse-health-effects upon abusive consumption (Dews, 1982). Caffeine is also an ideal probe to measure in vivo CYP1A2 activity (Doehmer et al., 1992; Miners et al., 1996). The pharmacokinetics of caffeine in humans has been studied extensively (Kot and Daniel, 2008). The "perpetrators" in the present study are the linear furanocoumarins (Figure 1) which are chemical isomers and congeners found in Apiaceae, Leguminosae, Moraceae, and Rutaceae plant families (Diawara and Trumble, 1997). Previous in vitro liver microsomal studies have shown that the main metabolic pathway of 8-MOP and 
5-MOP is the oxidative ring-opening of the furan structure to form an epoxide, or an intermediate electrophilic reactive metabolite, which binds covalently to human CYP proteins (Fouin-Fortunet et al., 1986; Tinel et al., 1987; Mays et al., 1990; John et al., 1992). Other linear furanocoumarins such as ISOP (Kang et al., 2011) and psoralen (Zhuang et al., 2013) also have been shown to be TDI, or MBI, in human liver microsomes (HLM) or recombinant human CYP1A2 expressed in yeast.

Our previous studies have shown that detectable levels of 8-MOP, 5-MOP, and ISOP in nine traditional herbal medicine (Alehaideb et al., 2017). We also have shown four of the furanocoumarin-containing herbs significantly reduced the oral clearance of caffeine in human volunteers (Alehaideb et al., 2021). Furthermore, the aforementioned furanocoumarins were found to be potent TDI or MBI of CYP1A2 isozyme (Alehaideb et al., 2021). The objectives of this study were: (1) to modify the predictive DDI models of Mayhew et al. and Wang et al. for in vivo herb-caffeine interaction using in vitro caffeine metabolism data in HLM and in vivo $\mathrm{C}_{\max }$ of furanocoumarins in humans, and (2) to determine the accuracy of Mayhew et al. and Wang et al. DDI predictive models by comparing predicted caffeine AUCR with experimental AUCR from Alehaideb et al. (2021).

\section{Methods}

\subsection{Source of plant products}

The plant products used in this study were obtained from North American commercial suppliers as follows: Ammi majus L. (A. majus) purchased from EverWilde (Fallbrook, CA), Angelica archangelica L. seeds (A. archangelica), A. graveolens seeds (A. 
graveolens S), Pimpinella anisum L. seeds (P. anisum), and Ruta graveolens L. leaves (R. graveolens) were purchased from Mountain Rose (Eugene, OR). Apium graveolens L. leaves (A. graveolens L) and Petroselinum crispum (Mill.) Fuss leaves (P. crispum) were purchased from A1SpiceWorld (Glen Head, NY). Angelica pubescens Maxim. roots (A. pubescens) purchased from Spring Wind (San Francisco, CA), Cnidium monnieri (L.) Cusson (C. monnieri) were purchased from Health and Wellness House (Duncan, BC). The plant products were further authenticated chromatographically as detailed in our previous publication (Alehaideb et al., 2017).

\subsection{Using DDI models to predict herb-caffeine interactions.}

In the present study, caffeine is the victim-drug and the furanocoumarin bioactive in the herbs are the perpetrators or inhibitors as mentioned earlier. As both caffeine and furanocoumarin inhibitors are metabolized by the same CYP1A2 enzyme, metabolic inhibition may occur in humans after co-administration. Indeed, the furanocoumarins have been shown previously to be TDI of CYP1A2. We have used two different DDI models to predict the inhibition of caffeine metabolism by furanocoumarin-containing herbs namely the Mayhew et al. and Wang et al. DDI models.

\subsubsection{Using Mayhew et al. model to predict caffeine-herb interactions.}

Mayhew et al. first proposed a DDI model involving the destruction of CYP enzyme by the reactive metabolite(s) of the inhibitor or the mechanism-based DDI model as follows:

Equation 1: The IVIVE DDI prediction model of Mayhew et al. (2000). 


$$
\text { AUCR }=\frac{\text { AUC }_{\mathrm{I}}}{\text { AUC }}=\frac{1}{1+\left(\frac{\mathrm{f}_{\mathrm{m}}}{\frac{[\mathrm{I}]_{\mathrm{H}} \times \mathrm{k}_{\text {inact }}}{\mathrm{k}_{\mathrm{deg}} \times\left([\mathrm{I}]_{\mathrm{H}}+\mathrm{K}_{\mathrm{I}}\right)}}\right)+\left(1-\mathrm{f}_{\mathrm{m}}\right)}
$$

where AUC is the area-under-curve of caffeine with no herbal extract pre-treatment, $\mathrm{AUC}_{\mathrm{I}}$ is the area-under-curve of caffeine with herbal extract pre-treatment, $\mathrm{k}_{\mathrm{deg}}$ is the first-order in vivo degradation rate constant for CYP enzyme, $\mathrm{K}_{\mathrm{I}}$ represents the equilibrium dissociation constant for the inactivator, $\mathrm{k}_{\text {inact }}$ is the maximum rate of enzyme inactivation at saturating concentrations of inhibitor, $f_{m}$ is the fraction of metabolic pathway, and $[\mathrm{I}]_{\mathrm{H}}$ represents the in vivo hypothetical intra-hepatic concentration(s) of the integrated dose/concentration of furanocoumarin inhibitors. Thus, the model of Mayhew et al. required the determination of $\mathrm{K}_{\mathrm{I}}, \mathrm{k}_{\text {inact }}, \mathrm{k}_{\mathrm{deg}}$ and $[\mathrm{I}]_{\mathrm{H}}$ for the chemical marker $(\mathrm{CM})$ furanocoumarin of 8-MOP only.

The theoretical background of mechanism-based model has been described in great detail elsewhere (Mayhew et al., 2000). The model is able to predict quantitatively the inhibition of drugs if the following conditions are met: (a) the well-stirred liver conditions are met, (b) the in vitro inhibition constants are applicable to in vivo interaction, and (c) the $[\mathrm{I}]_{\mathrm{H}}$ value is lower than the $\mathrm{K}_{\mathrm{I}}$ inhibition constant value.

An integrated dose/concentration of the furanocoumarin mixture in the liver, $[\mathrm{I}]_{\mathrm{H}}$ was predicted using the CA model (Safe, 1998; ATSDR, 2004; Alehaideb et al., 2019) with 8-MOP as the CM as follows:

Equation 2: The concentration-addition (CA) model approach.

$$
\text { Furanocoumarin CM equivalent concentration }=\sum \mathrm{CI} \times \mathrm{RPF}
$$


where $\mathrm{CI}$ is the mass concentration of the individual furanocoumarin in the chemical mixture and RPF is the relative potency of each chemical in comparison to 8-MOP potency.

The CA model is applicable to the present study because: (a) the furanocoumarin inhibitors are chemical isomers and congeners that exhibit similar biological mechanisms, (b) the dose-inhibition curves of individual furanocoumarins are parallel to one another as the slopes of the dose-inhibition curves of 8-MOP, 5-MOP, and ISOP are $2.0,1.6$, and 1.9 respectively, and (c) the inhibitory potencies of individual furanocoumarins are additive in nature.

\subsubsection{Using Wang et al. model to predict caffeine-herb interaction.}

Instead of calculating a single $[\mathrm{I}]_{\mathrm{H}}$ with the CA model (Safe, 1998; ATSDR, 2004), individual furanocoumarin $[\mathrm{I}]_{\mathrm{H}}$ were calculated separately before adding them together to yield the AUCR. Thus, Wang et al. model required the determination of the $\mathrm{K}_{\mathrm{I}}$, $\mathrm{k}_{\text {inact, }}$, $\mathrm{k}_{\mathrm{deg}}$, and $[\mathrm{I}]_{\mathrm{H}}$ values for each individual furanocoumarin inhibitor as follows:

Equation 3: The IVIVE DDI prediction model of Wang et al. (2004).

$$
\text { AUCR }=\frac{\text { AUC }_{I}}{A U C}=\frac{1}{1+\left(\sum \frac{f_{m}}{\frac{[I]_{H} \times k_{\text {inact }}}{k_{\text {deg }} \times K_{I}}}\right)+\left(1-f_{m}\right)}
$$

Since caffeine undergoes negligible first-pass metabolism (Kalow and Tang, 1993), the DDI model of Wang et al. was modified for the present study by excluding the intestinal metabolism term from the original equation.

Figure 1 summarizes the experimental procedure and validation steps of the herbcaffeine interaction studies. Model-predicted AUCR was compared with experimental 
AUCR in the previous pharmacokinetic studies (Alehaideb, 2021) in order to validate the IVIVE models for herb-caffeine interaction prediction.

\subsubsection{IVIVE DDI model input parameters.}

The predictive models of Mayhew et al. and Wang et al. used a combination of in vitro caffeine metabolism inhibition parameters (Alehaideb et al., 2021) and in vivo furanocoumarin $[\mathrm{I}]_{\mathrm{H}}$ concentrations to predict quantitative impairment of in vivo caffeine clearance due to co-administration of caffeine and furanocoumarin-containing herbs. As shown in equations 1 and 2, in vivo caffeine clearance impairment does not depend on the concentration of caffeine but is dependent on the concentrations of furanocoumarin inhibitors at the site of metabolism, the liver. As the intra-hepatic concentrations of furanocoumarin inhibitors could not be determined by direct experiment, four different furanocoumarin dose surrogates (i.e., maximum total plasma concentration $\left(\mathrm{C}_{\mathrm{max}, \mathrm{PT}}\right)$, maximum unbound plasma concentration $\left(\mathrm{C}_{\mathrm{max}, \mathrm{PU}}\right)$, maximum total liver concentration level $\left(\mathrm{C}_{\mathrm{max}, \mathrm{LT}}\right)$, and maximum unbound liver concentration $\left(\mathrm{C}_{\max , \mathrm{LU}}\right)$ were used to calculate the $[\mathrm{I}]_{\mathrm{H}}$ for the predictive models.

\subsubsection{In vitro caffeine metabolism inhibition data.}

The in vitro experimental inhibition parameters we obtained using radiolabeled caffeine and pooled human liver microsomes as described in detail in our previous study (Alehaideb et al., 2021). Briefly, the $\mathrm{IC}_{50}$ values were obtained using serial dilutions of pure furanocoumarin incubated with radiolabeled caffeine, microsomes, and NADPH cofactor. The radiolabeled metabolites were collected by solid-phase extraction and counted by scintillation. The inactivation constants were experimentally measured using the two-step dilution with preincubation time points from 0.5 to $4 \mathrm{~min}$. The $\mathrm{IC}_{50}$ 
of 8-MOP, 5-MOP, and ISOP were $0.09,0.13$, and $0.29 \mu \mathrm{M}$, respectively; the corresponding $\mathrm{K}_{\mathrm{I}}$ values were $0.78,3.73$, and $4.48 \mu \mathrm{M}$, and $\mathrm{k}_{\text {inact }}$ values were $0.17,0.35$, and $0.65 \mathrm{~min}^{-1}$, respectively (Alehaideb et al., 2021). The $\mathrm{k}_{\mathrm{deg}}$ value of CYP1A2, $0.0003 \mathrm{~min}^{-1}$, was obtained from Faber and Fuhr (2004). The $\mathrm{f}_{\mathrm{m}}$ value for caffeine in humans, 0.95, was taken from Kalow and Tang (1993). Thus, only the $\mathrm{C}_{\text {max }}$-derived $[\mathrm{I}]_{\mathrm{H}}$ remained to be determined in the IVIVE models.

\subsubsection{Furanocoumarin $C_{\max }$ extrapolation based on dose- $C_{\max }$ relationship.}

Table 1 lists the body weights (BW) of the volunteers and the doses of individual furanocoumarins administered as described previously in our publication (Alehaideb et al., 2017). These furanocoumarin doses were used as the administered doses in predicting the $\mathrm{C}_{\text {max,PT }}$ of 5-MOP and 8-MOP in the plasma of humans based on allometric extrapolation (Gabrielsson and Weiner, 2006). The $\mathrm{C}_{\max , \mathrm{PT}}$ of ISOP was the average of the 5-MOP and 8-MOP values. The following was a detailed description of predicting $\mathrm{C}_{\max , \mathrm{PT}}, \mathrm{C}_{\max , \mathrm{PU}}, \mathrm{C}_{\max , \mathrm{LT}}$, and $\mathrm{C}_{\max , \mathrm{LU}}$ concentration levels in humans:

(a) The $\mathrm{C}_{\text {max,PT }}$ of 8-MOP and 5-MOP were obtained by allometric extrapolation from the experimental data of Schfifer-Korting and Korting (1982) and Stolk et al. (1981) studies, respectively. Figure $2 \mathrm{a}$ shows the administered dose versus serum $\mathrm{C}_{\max , \mathrm{PT}}$ plot of 8-MOP (Schfifer-Korting and Korting, 1982). Figure 2b shows the administered dose versus serum $\mathrm{C}_{\text {max,PT }}$ plot of 5-MOP (Stolk et al. 1981). Despite our best effort, we were unable to find any animal or human data for proper allometric extrapolation of ISOP. Instead, we assumed the $\mathrm{C}_{\max , \mathrm{PT}}$ values of ISOP to be the average of 8-MOP and 5-MOP extrapolated values. 
(b) $\mathrm{C}_{\mathrm{max}, \mathrm{PU}}$ was derived from $\mathrm{C}_{\mathrm{max}, \mathrm{PT}}$ by multiplying the latter with the unbound fractions (fup) of 8-MOP or 5-MOP in the plasma; they were $17.0( \pm 7.4)$ and $3.6( \pm 2.2)$ percent respectively (Veronese et al., 1978; Artuc et al., 1979; Pibouin et al., 1987; Makki et al., 1991; Muret et al., 1993a). No information was found for ISOP. Therefore, an average of 8-MOP and 5-MOP values was used.

(c) The $\mathrm{C}_{\max , \mathrm{LT}}$ was derived by multiplying $\mathrm{C}_{\max , \mathrm{PT}}$ with the liver:plasma partition coefficient (Pt:p) which was calculated as follows:

Equation 4: Non-adipose tissue:plasma partition model of Poulin and Theil (2002).

$$
\mathrm{P}_{\mathrm{t}: \mathrm{p}}=\frac{\mathrm{P}_{\mathrm{o} / \mathrm{w}} \times\left(\mathrm{V}_{\mathrm{nt}}+0.3 \times \mathrm{V}_{\mathrm{pht}}\right)+\left(\mathrm{V}_{\mathrm{wt}}+0.7 \times \mathrm{V}_{\mathrm{pht}}\right)}{\mathrm{P}_{\mathrm{o} / \mathrm{w}} \times\left(\mathrm{V}_{\mathrm{np}}+0.3 \times \mathrm{V}_{\mathrm{php}}\right)+\left(\mathrm{V}_{\mathrm{wp}}+0.7 \times \mathrm{V}_{\mathrm{php}}\right)} \times \frac{\mathrm{f}_{\mathrm{up}}}{\mathrm{f}_{\mathrm{ut}}}
$$

where $\mathrm{P}_{\mathrm{o} / \mathrm{w}}$ is the $n$-octanol/water partition for non-ionized inhibitor, $\mathrm{V}_{\mathrm{nt}}$ is the fraction weight of neutral lipids in liver tissue, $\mathrm{V}_{\text {pht }}$ is the fraction weight of phospholipids in liver tissue, $\mathrm{V}_{\mathrm{wt}}$ is the fraction weight of water in liver tissue, $\mathrm{V}_{\mathrm{np}}$ is the fraction weight of neutral lipids in plasma, $\mathrm{V}_{\mathrm{wp}}$ is the fraction weight of water in plasma, $\mathrm{V}_{\mathrm{php}}$ is the fraction weight of phospholipids in plasma, $\mathrm{f}_{\mathrm{up}}$ is the fraction unbound in plasma, and $f_{u t}$ is the fraction unbound in liver tissue.

The $\mathrm{P}_{\mathrm{o} / \mathrm{w}}$ of 8-MOP, 5-MOP, and ISOP were $120.2,128.8$, and 169.8 respectively; they were obtained from the Advanced Chemistry Development I-lab databases (ACD, 2015). The values of $\mathrm{V}_{\mathrm{nt}}, \mathrm{V}_{\mathrm{pht}}, \mathrm{V}_{\mathrm{wt}}, \mathrm{V}_{\mathrm{np}}, \mathrm{V}_{\mathrm{wp}}$, and $\mathrm{V}_{\mathrm{php}}$ were $0.035,0.025,0.751,0.004$, 0.945, and 0.002 respectively; they were also obtained from Poulin and Theil (2000).

(d) The $\mathrm{C}_{\max , \mathrm{Lu}}$ was derived by multiplying the $\mathrm{C}_{\max , \mathrm{LT}}$ by the calculated unbound fraction $\left(f_{u t}\right)$ in tissue. The $f_{u t}$ was determined using the following equation:

Equation 5: Unbound tissue fraction model of Poulin and Theil (2000). 


$$
f_{u t}=\frac{1}{1+\left(\left(\frac{1-f_{u p}}{f_{u p}}\right) \times 0.5\right)}
$$

\subsection{Human caffeine pharmacokinetic studies}

The in vitro pharmacokinetic data for caffeine metabolism inhibition due to consumption of furanocoumarin-containing herbal products were reported in our previous publications in great detail (Alehaideb et al., 2021). Briefly, eligible volunteers were dosed with $200 \mathrm{mg}$ caffeine twice: with and without prior treatment with an aqueous extract of one selected herbal medicine. Saliva samples were collected at timepoints ranging between 0 to 48 hours. Caffeine and internal standard were separated and measured chromatographically using an isocratic method with an ultra-violet detector. The salivary caffeine concentrations were converted into plasma concentrations using a conversion factor of 0.79 . The human plasma caffeine AUC from zero to infinity $\left(\mathrm{AUC}_{0 \text {-inf }}\right)$ for each volunteer was measured twice and the experimental AUCR was calculated using the PKsolver software.

\subsection{Data and statistical analysis.}

Data plotting and extrapolation were performed using GraphPad Prism Software version 5.04 (San Diego, CA). Statistical analysis was performed using Microsoft Excel software. Model-predicted AUCR were reported as mean \pm standard deviation (SD). Herb-caffeine interaction occurred when the mean AUCR was equal or greater than 2.0. No interaction occurred when the mean AUCR was less than 2.0 (Einolf, 2007). The geometric mean-fold error (GMFE) (Equation 6) was also used to assess the accuracy of model prediction by equal weighting under-predictions and over-predictions. The 
model that predicted perfectly would give a GMFE value of 1; GMFE value between 1 and 2-fold is considered to be accurate.

Equation 6: The geometric mean-fold error (GMFE) (Obach et al., 1997).

$$
\text { GMFE }=10^{\sum\left|\log \frac{\text { Predicted HDI }}{\text { Actual HDI }}\right|}
$$

where $\mathrm{n}$ is the number of predictions for each herb.

\section{Results and Discussion}

Tables 2 to 5 show the experimental and model-predicted AUCR of caffeine in humans as a result of pre-treatment with an herbal extract. The variability of the experimental AUCR is large but consistent with the large variation of 8-MOP (Herfst and De Wolff, 1982) and 5-MOP (Ehrsson et al., 1994) concentrations observed in the serum. Relatively high experimental AUCR were observed in volunteers pre-treated by $A$. majus, A. archangelica, A. pubescens, C. monnieri, or R. graveolens (Table 2 to 5). These results are consistent with the relatively high levels of furanocoumarins found in the herbal extracts (Table 1). Moreover, the presence of osthole, a CYP1A2 inhibitor (Yang et al., 2012), in A. pubescens may contribute to the high AUCR of this herbal extract. Similarly, the relatively high AUCR of C. monnieri may be due to osthole in addition to high levels of 8-MOP, 5-MOP, and ISOP. Despite only low levels of furanocoumarins have been found in P. crispum; mean AUCR in the volunteers is noticeable. This may be due to the presence of flavones such as apigenin in the extract (Meyer et al., 2006), which is a CYP1A2 inhibitor (Peterson et al., 2006).

Wang et al. IVIVE model predicted $a \geq 2$-fold increase in mean caffeine AUCR after pre-treating the volunteers with $A$. majus seeds or $C$. monnieri fruits extract regardless 
of $\mathrm{C}_{\max }$ used to derive the $[\mathrm{I}]_{\mathrm{H}}$ value. However, the interaction due to A. archangelica roots pretreatment was not predicted successfully using $\mathrm{C}_{\max , \mathrm{PU}}$ values (Table 3 ). The AUCR derived from $\mathrm{C}_{\max , \mathrm{PU}}$ and $\mathrm{C}_{\mathrm{max}, \mathrm{LU}}$ appeared to have fewer false positive results in the remaining 6 herbs on the list (Tables 2 to 5). The average GMFE for $\mathrm{C}_{\max , \mathrm{PU}}$ and $\mathrm{C}_{\text {max,Lu were }} 1.7$ and 2.0 (Tables 3 and 5) indicating $\mathrm{C}_{\max , \mathrm{PU}}$ and $\mathrm{C}_{\max , \mathrm{LU}}$ yield more accurate AUCR results than $\mathrm{C}_{\max , \mathrm{PT}}$ and $\mathrm{C}_{\max , \mathrm{LT}}$ which had average GMFE value of 2.6 and 3.5 respectively (Tables 2 and 4). These results are consistent with the larger number of false positive results in $\mathrm{C}_{\max , \mathrm{PT}}$ and $\mathrm{C}_{\max , \mathrm{LT}}$ (Tables 2 and 4). The actual AUCR versus predicted AUCR correlation plot confirms $\mathrm{C}_{\max , \mathrm{PU}}$ and $\mathrm{C}_{\mathrm{max}, \mathrm{LU}}$ are better dose surrogates to predict caffeine AUCR than $\mathrm{C}_{\max , \mathrm{PT}}$ and $\mathrm{C}_{\max , \mathrm{LT}}$ (Figure 4).

In contrast, when the IVIVE model of Mayhew et al. was used to predict the AUCR of caffeine in humans pre-treated by herbal extracts, only $\mathrm{C}_{\max , \mathrm{LT}}$ predicted the experimental AUCR closely (Table 4). An exception was $R$. graveolens leaves which overpredicted the experimental AUCR. The average GMFE were similar for $\mathrm{C}_{\max , \mathrm{LU}}$ $\mathrm{C}_{\max , \mathrm{PT}}$ and $\mathrm{C}_{\text {max,PU }}$ at 1.7 or 1.8 (Tables 2 to 5). However, $\mathrm{C}_{\max , \mathrm{PU}}$ and $\mathrm{C}_{\max , \mathrm{LU}}$ generally underpredicted the experimental AUCR with the exception of A. majus seeds which was always overpredicted regardless of the DDI model used. Thus, $\mathrm{C}_{\max , \mathrm{LT}}$ yielded the most accurate prediction for caffeine metabolism inhibition with average GMFE of 1.8 (Table 4). The actual AUCR versus predicted AUCR correlation plot also confirms $\mathrm{C}_{\max , \mathrm{LT}}$ is a better dose surrogate to predict caffeine AUCR than $\mathrm{C}_{\max , \mathrm{LU}}, \mathrm{C}_{\max , \mathrm{PT}}$ and $\mathrm{C}_{\max , \mathrm{PU}}($ Figure 4).

Despite no agreement is reached on the most accurate $[\mathrm{I}]_{\mathrm{H}}$ surrogate for DDI predictions, previous studies have reported accurate DDI prediction by using either total $\mathrm{C}_{\max }$ or unbound $\mathrm{C}_{\max }$ in the liver and plasma to estimate $[\mathrm{I}]_{\mathrm{H}}$ (Grimm et al., 2009). For 
example, Ito et al. $(2004 ; 2005)$ have reported that $\mathrm{C}_{\max , \mathrm{LT}}$ yields the most accurate prediction for reversible-based inhibition DDI. This is consistent with the suggestion of Brown et al. (2006) that incorporating protein binding into the predictive model did not improve reversible-based DDI prediction. In contrast, Obach et al. (2006) and Fahmi et al. (2009) have concluded that $\mathrm{C}_{\max , \mathrm{LU}}$ is most accurate in reversible-based DDI prediction. Blanchard et al. (2004) have reported $\mathrm{C}_{\max , \mathrm{PU}}$ provides the most accurate DDI predictions based on reversible inhibition. For irreversible-based inhibition predictions, Obach et al. (2007) and Fahmi et al. (2009) have suggested $\mathrm{C}_{\mathrm{max}, \mathrm{PU}}$ is the most accurate dose surrogate for DDI prediction. In contrast, both Ito et al. (2003) and Shardlow et al. (2011) reported $C_{\max , L u}$ provided the most accurate DDI predictions.

Although both Wang et al. and Mayhew et al. models were able to predict the empirical AUCR of caffeine, Wang et al. model is more predictive than Mayhew et al. model because the latter is implemented with a single, composite $[\mathrm{I}]_{\mathrm{H}}$ value which is less accurate than a combination of individual $[\mathrm{I}]_{\mathrm{H}}$ values in Wang et al. model. The predictability of the IVIVE model is also affected by the kinetic parameters as follows: (a) the $\mathrm{k}_{\text {deg }}$ value of hCYP1A2. The $0.00030 \mathrm{~min}^{-1} \mathrm{k}_{\text {deg }}$ used in the present study is based on the $38 \mathrm{~h} \mathrm{t}_{1 / 2}$ of a tobacco smoking cessation study (Faber and Fuhr, 2004). Mayhew et al. has reported a $\mathrm{k}_{\mathrm{deg}}$ value of $0.00083 \mathrm{~min}^{-1}$ which is derived from rats. If this $\mathrm{k}_{\mathrm{deg}}$ was used in the present study, both predictive models would underestimate the AUCR of caffeine, (b) in vitro kinetic inhibition parameters such as $\mathrm{IC}_{50}, \mathrm{~K}_{\mathrm{I}}$ and $\mathrm{k}_{\text {inact }}$ were derived using pooled HLM from multiple donors. This also appears to improve the accuracy of our predictions, and (c) caffeine as the victim drug has simplified model prediction by eliminating the need to account for parallel metabolic pathways by other 
CYP isoforms and urinary excretion of unchanged caffeine. As a result, the uncertainty involved in the AUCR calculation is greatly reduced.

The following are some of the uncertainties or limitations of the present study: (a) the data used to establish the 8-MOP dose-response curves for allometric extrapolation are based on psoriasis patients and these raised concern that plasma 8-MOP levels in healthy and psoriasis subjects might be different. However, Shephard et al. (1999) has shown that similar free 8-MOP fractions are found in the plasma of psoriasis and healthy volunteers. Also, Muret et al. (1993b) have shown that the fractions of free 5MOP in the serum of healthy and psoriasis volunteers do not differ significantly. Together, these results suggest that systemic 8-MOP and 5-MOP levels do not differ significantly in healthy and psoriasis subjects, (b) in the present study, the pharmacokinetic values of individual furanocoumarins in an herbal extract and pure furanocoumarins are assumed to be similar. This assumption might be incorrect since pharmacokinetic interaction between individual furanocoumarins in an herbal extract may occur, and (c) previous studies have shown that both 8-MOP (Mays et al., 1987; Apseloff et al., 1990) and ISOP (Baumgart et al., 2005) induce hCYP1A2 enzyme activity in rats after multiple dosing. In contrast, Tantcheva-Poór et al. (2001) dosed humans with $0.6 \mathrm{mg}$ of $8-\mathrm{MOP} / \mathrm{kg} / \mathrm{day}$ and found no significant change in caffeine clearance even after a week of treatment. In the present study, the inductive effect of 8MOP and ISOP is ignored as only a single dose of herbal extract is administered $3 \mathrm{~h}$ before the caffeine pharmacokinetic studies, and it is unlikely hCYP1A2 can be induced with a short pretreatment period. 


\section{Conclusions}

Using IVIVE models to predict HDI is an ongoing research program in our laboratories. Our goal is to develop a reliable and simple prediction tool for HDI. Results of this study suggest that linear furanocoumarins such as 8-MOP, 5-MOP and ISOP are responsible for the inhibition of caffeine metabolism in humans after consuming herbal extracts containing furanocoumarin derivatives. The described modeling approaches in this study may also be applicable to health supplements and functional food.

\section{Acknowledgements}

We thank Dr. Sabine Matou-Nasri (King Abdullah International Medical Research Center (KAIMRC), Riyadh city, Kingdom of Saudi Arabia) and Professor Francis Law (Simon Fraser University, Burnaby city, British Columbia, Canada) for providing comments and suggestions that improved this manuscript.

\section{Funding}

This work received no external funding.

\section{Conflict of Interest}

The authors declare that there are no conflicts of interest.

\section{Author Contribution}

All authors have contributed equally. 


\section{List of Figures}

Figure 1: Flow-chart summary of experimental procedures and validation steps in the use of drug-drug interaction models to predict herb-drug interaction.

Figure 2: Ln-Ln plot of (a) 8-MOP dose versus $\mathrm{C}_{\text {max,PT }}$ data from Schfifer-Korting and Korting (1982) and (b) Ln-Ln plot of 5-MOP dose versus $\mathrm{C}_{\max , \mathrm{PT}}$ data from Stolk et al. (1981).

Figure 4: Correlation of (a) Wang et al. (2004) model-predicted AUCR and (b) of Mayhew et al. (2000) model-predicted AUCR against actual AUCR using different $[\mathrm{I}]_{\mathrm{H}}$ surrogate values. 


\section{List of Tables}

Table 1: A summary of BW and individual furanocoumarin doses for human volunteers in the caffeine pharmacokinetic studies.

Table 2: A comparison of model-predicted $\mathrm{C}_{\max , \mathrm{PT}-\text { based AUCR and experimental }}$ AUCR in humans due to herbal extract pretreatment. IVIVE model was modified from Wang et al. (2004).

Table 3: A comparison of model-predicted $\mathrm{C}_{\mathrm{max}, \mathrm{PU}}$-based AUCR and experimental AUCR in humans due to herbal extract pretreatment. IVIVE model was modified from Wang et al. (2004).

Table 4: A comparison of model-predicted $\mathrm{C}_{\max , \mathrm{LT}}$-based AUCR and experimental AUCR in humans due to herbal extract pretreatment. IVIVE model was modified from Wang et al. (2004).

Table 5: A comparison of model-predicted $\mathrm{C}_{\max , \mathrm{LU}}$-based AUCR and experimental AUCR in humans due to herbal extract pretreatment. IVIVE model was modified from Wang et al. (2004). 


\section{List of Equations}

Equation 1: The IVIVE DDI prediction model of Mayhew et al. (2000).

Equation 2: The concentration-addition (CA) model approach.

Equation 3: The IVIVE DDI prediction model of Wang et al. (2004).

Equation 4: Non-adipose tissue:plasma partition model of Poulin and Theil (2002).

Equation 5: Unbound tissue fraction model of Poulin and Theil (2000).

Equation 6: The geometric mean-fold error (GMFE) (Obach et al., 1997). 
Figure 1<smiles>O=c1ccc2cc3ccoc3cc2o1</smiles>

Psoralen<smiles>COc1c2ccoc2c(OC)c2oc(=O)ccc12</smiles>

Isopimpinellin (ISOP)<smiles>COc1c2occc2cc2ccc(=O)oc12</smiles>

8-methoxypsoralen (8-MOP)<smiles>COc1c2ccoc2cc2oc(=O)ccc12</smiles>

5-methoxypsoralen (5-MOP) 


\section{Figure 2}

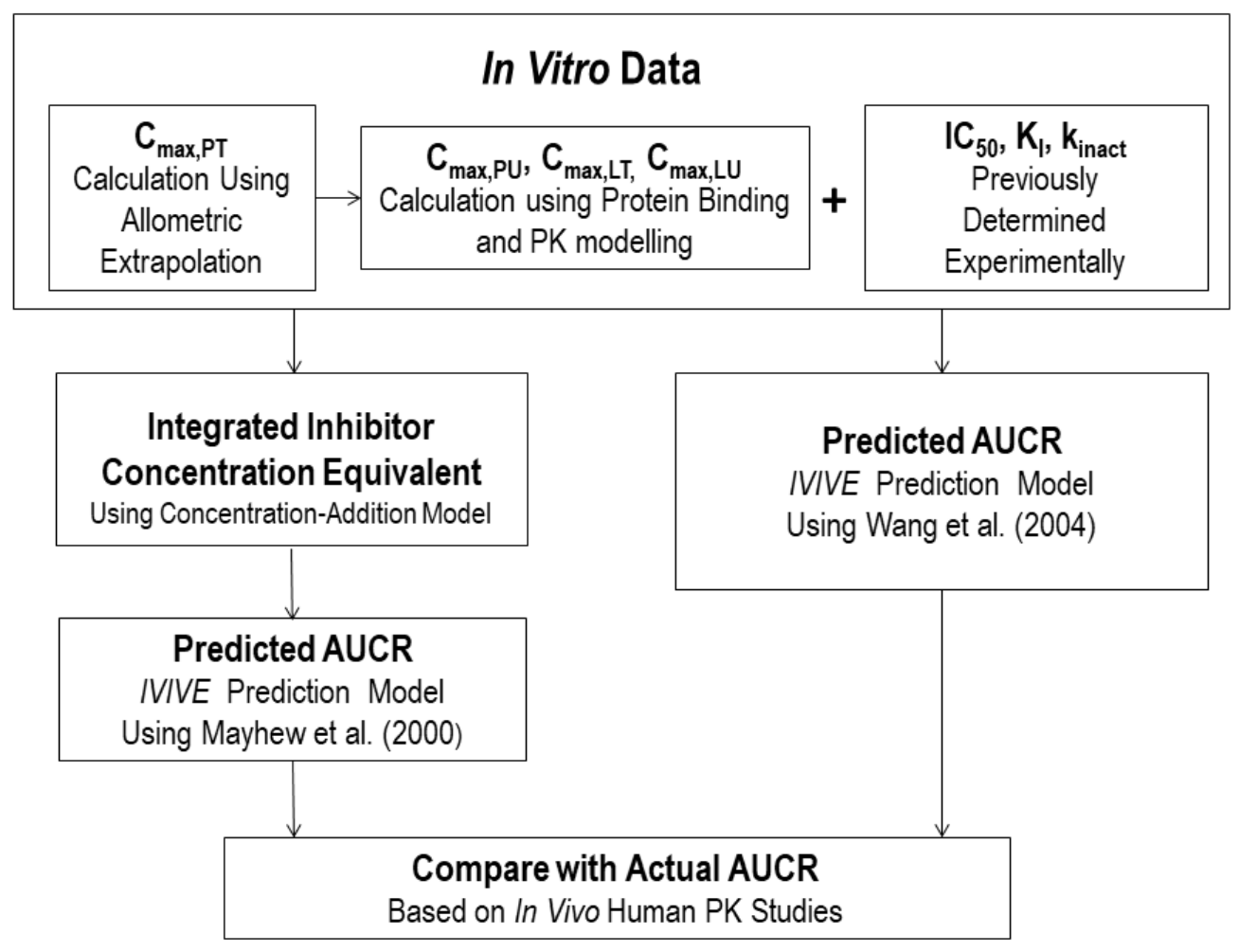


Figure 3
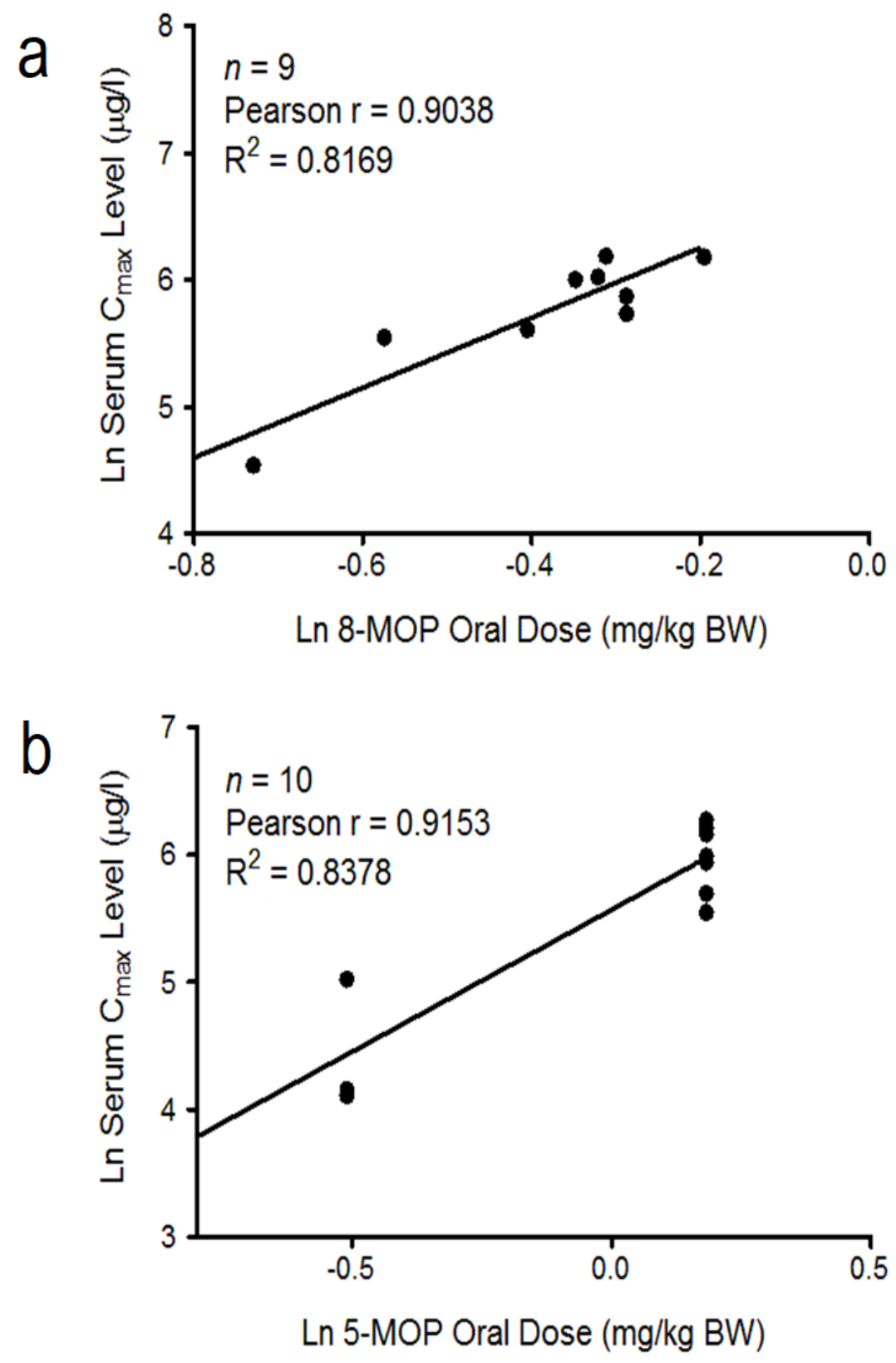
Figure 4

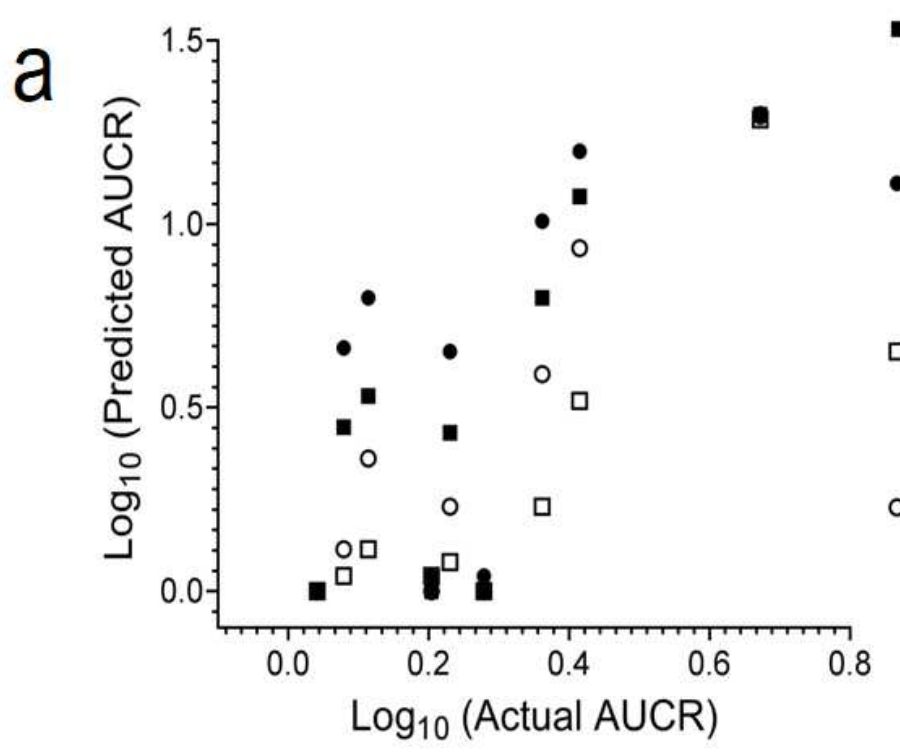

- $\mathrm{C}_{\text {max,PT }}$

Pearson $r=0.7771$

$\mathrm{R}$ square $=0.6039$

- $\mathrm{C}_{\max , \mathrm{LT}}$

Pearson $r=0.6684$

$\mathrm{R}$ square $=0.4468$

- $\mathrm{C}_{\max , \mathrm{PU}}$

Pearson $r=0.8946$

$\mathrm{R}$ square $=0.8004$

- $\mathrm{C}_{\max , \mathrm{LU}}$

Pearson $r=0.8727$

$\mathrm{R}$ square $=0.7617$

b

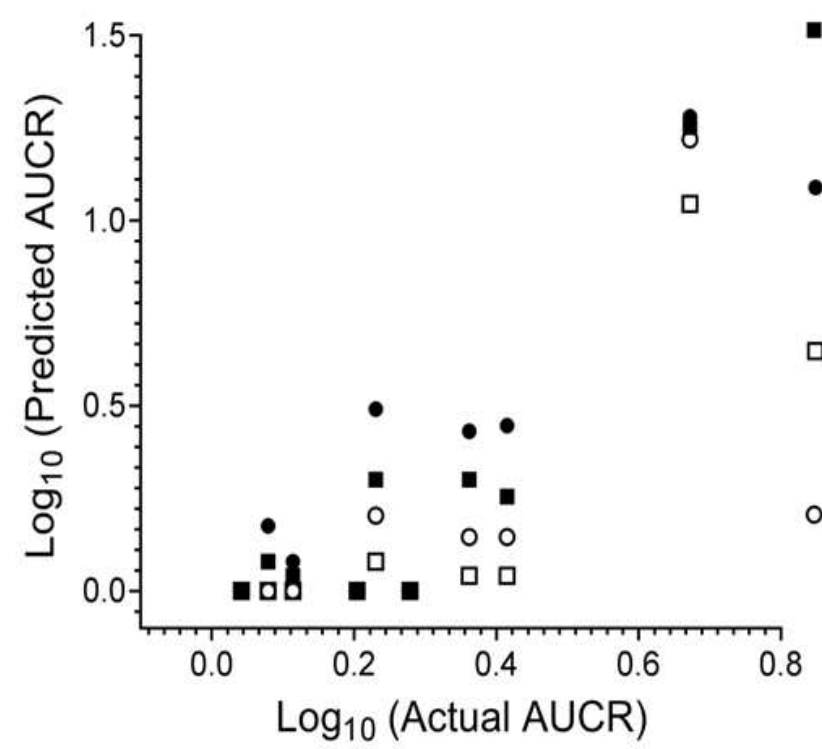

- $\mathrm{C}_{\text {max,PT }}$

Pearson $r=0.8653$

$\mathrm{R}$ square $=0.7488$

- $\mathrm{C}_{\max , \mathrm{LT}}$

Pearson $r=0.8676$

$\mathrm{R}$ square $=0.7528$

- $\mathrm{C}_{\max , \mathrm{PU}}$

Pearson $r=0.7991$

$\mathrm{R}$ square $=0.6385$

- $\mathrm{C}_{\max , \mathrm{LU}}$

Pearson $r=0.8424$

$\mathrm{R}$ square $=0.7096$ 


\section{Table 1}

\begin{tabular}{|c|c|c|c|c|c|c|c|c|c|c|c|c|c|}
\hline \multirow{4}{*}{$\begin{array}{l}\text { Plant Name and Part } \\
\text { A. majus seeds }\end{array}$} & \multicolumn{13}{|c|}{ Human Furanocoumarin Oral Dose } \\
\hline & \multirow{3}{*}{$\begin{array}{l}n \\
4\end{array}$} & \multicolumn{3}{|c|}{ BW a } & \multicolumn{3}{|c|}{ 8-MOP a } & \multicolumn{3}{|c|}{ 5-MOP a } & \multicolumn{3}{|c|}{ ISOP a } \\
\hline & & \multicolumn{3}{|c|}{$\mathrm{kg}$} & \multicolumn{3}{|c|}{$\mu \mathrm{g} / \mathrm{kg} \mathrm{BW}$} & \multicolumn{3}{|c|}{$\mu \mathrm{g} / \mathrm{kg} \mathrm{BW}$} & \multicolumn{3}{|c|}{$\mu \mathrm{g} / \mathrm{kg} \mathrm{BW}$} \\
\hline & & 77.5 & \pm & 17.1 & 260.89 & \pm & 73.37 & 58.19 & \pm & 16.36 & 611.91 & \pm & 172.1 \\
\hline A. archangelica roots & 5 & 73.2 & \pm & 17.6 & 42.2 & \pm & 11.2 & 25.5 & \pm & 6.8 & 39.3 & \pm & 10.4 \\
\hline C. monnieri fruits & 5 & 75.8 & \pm & 7.4 & 28.2 & \pm & 3 & 71.3 & \pm & 7.5 & 18.6 & \pm & 2 \\
\hline$R$. graveolens leaves & 4 & 72.0 & \pm & 6.9 & 56.3 & \pm & 5.7 & 22.4 & \pm & 2.3 & 12.3 & \pm & 1.3 \\
\hline A. pubescens roots & 4 & 75.0 & \pm & 8.2 & 4.1 & \pm & 0.5 & 5.2 & \pm & 0.6 & \multicolumn{3}{|c|}{ n.d. $b$} \\
\hline A. graveolens seeds & 4 & 79.3 & \pm & 5.8 & 2.7 & \pm & 0.2 & 2.1 & \pm & 0.1 & 29.9 & \pm & 2.1 \\
\hline A. graveolens Leaves & 2 & 81.5 & \pm & 3.5 & 1.5 & \pm & 0.1 & 29.9 & \pm & 1.3 & 1.2 & \pm & 0.1 \\
\hline P. crispum leaves & 2 & 81.5 & \pm & 3.5 & \multicolumn{3}{|c|}{ n.d. ${ }^{b}$} & 4.2 & \pm & 0.2 & \multicolumn{3}{|c|}{ n.d. ${ }^{b}$} \\
\hline$P$. anisum seeds & 4 & 75.8 & \pm & 8.5 & 2.1 & \pm & 0.3 & \multicolumn{3}{|c|}{ n.d. $b$} & \multicolumn{3}{|c|}{ n.d. $b$} \\
\hline
\end{tabular}

a Expressed as mean \pm SD.

${ }^{\mathrm{b}}$ n.d. = not detected. 


\section{Table 2}

\begin{tabular}{|c|c|c|c|c|c|c|c|c|c|c|c|c|c|}
\hline \multirow{3}{*}{ Botanical Name } & \multirow{2}{*}{\multicolumn{3}{|c|}{ Actual AUCR }} & \multicolumn{10}{|c|}{ Predicted AUCR } \\
\hline & & & & \multicolumn{5}{|c|}{ Wang et al. (2004) } & \multicolumn{5}{|c|}{ Mayhew et al. (2000) } \\
\hline & Mean & \pm & $S D$ & Mean & \pm & SD & Predictive a & GMFE b & Mean & \pm & SD & Predictive a & GMFE b \\
\hline A.majus seeds & 4.7 & \pm & 1.0 & 19.9 & \pm & 0.0 & + & 4.3 & 17.9 & \pm & 0.9 & + & 3.9 \\
\hline A.archangelica roots & 2.3 & \pm & 0.6 & 6.3 & \pm & 2.4 & + & 2.7 & 2.0 & \pm & 0.6 & + & 1.2 \\
\hline C.monnieri fruits & 2.6 & \pm & 1.2 & 11.9 & \pm & 1.1 & + & 5.0 & 1.8 & \pm & 0.2 & - & 1.5 \\
\hline R.graveolens leaves & 1.7 & \pm & 0.5 & 2.7 & \pm & 0.4 & - & 1.6 & 2.0 & \pm & 0.3 & - & 1.4 \\
\hline A.pubescens roots & 1.9 & \pm & 0.8 & 1.0 & \pm & 0.0 & + & 1.7 & 1.0 & \pm & 0.0 & + & 1.8 \\
\hline A.graveolens seeds & 1.3 & \pm & 0.7 & 3.4 & \pm & 0.3 & - & 2.8 & 1.1 & \pm & 0.0 & + & 1.4 \\
\hline A.graveolens leaves & 1.2 & \pm & 0.3 & 2.8 & \pm & 0.2 & - & 2.4 & 1.2 & \pm & 0.0 & + & 1.2 \\
\hline P.crispum leaves & 1.6 & \pm & 0.9 & 1.0 & \pm & 0.0 & + & 1.6 & 1.0 & \pm & 0.0 & + & 1.6 \\
\hline P.aniseum seeds & 1.1 & \pm & 0.2 & 1.0 & \pm & 0.0 & + & 1.2 & 1.0 & \pm & 0.0 & + & 1.2 \\
\hline & & & & & & & Average & 2.6 & & & & Average & 1.7 \\
\hline
\end{tabular}

a Based on the two-fold rule; (+) predictive and (-) not predictive.

b Geometric mean-fold error; ( $\leq 2)$ indicate accurate prediction and (>2) is less accurate. 


\section{Table 3}

\begin{tabular}{|c|c|c|c|c|c|c|c|c|c|c|c|c|c|}
\hline \multirow{3}{*}{ Botanical Name } & \multirow{2}{*}{\multicolumn{3}{|c|}{ Actual AUCR }} & \multicolumn{10}{|c|}{ Predicted AUCR } \\
\hline & & & & \multicolumn{5}{|c|}{ Wang et al. (2004) } & \multicolumn{5}{|c|}{ Mayhew et al. (2000) } \\
\hline & Mean & \pm & SD & Mean & \pm & SD & Predictive a & GMFE b & Mean & \pm & SD & Predictive a & GMFE b \\
\hline A.majus seeds & 4.7 & \pm & 1.0 & 20.0 & \pm & 0.0 & + & 4.3 & 19.1 & \pm & 0.4 & + & 4.1 \\
\hline A.archangelica roots & 2.3 & \pm & 0.6 & 10.2 & \pm & 2.9 & + & 4.4 & 2.7 & \pm & 1.0 & + & 1.4 \\
\hline C.monnieri fruits & 2.6 & \pm & 1.2 & 15.8 & \pm & 0.8 & + & 6.7 & 2.8 & \pm & 0.4 & + & 1.5 \\
\hline R.graveolens leaves & 1.7 & \pm & 0.5 & 4.5 & \pm & 0.8 & - & 2.7 & 3.1 & \pm & 0.6 & - & 1.9 \\
\hline A.pubescens roots & 1.9 & \pm & 0.8 & 1.1 & \pm & 0.0 & + & 1.6 & 1.0 & \pm & 0.0 & + & 1.8 \\
\hline A.graveolens seeds & 1.3 & \pm & 0.7 & 6.3 & \pm & 0.6 & - & 5.2 & 1.2 & \pm & 0.0 & + & 1.4 \\
\hline A.graveolens leaves & 1.2 & \pm & 0.3 & 4.6 & \pm & 0.3 & - & 3.8 & 1.5 & \pm & 0.0 & + & 1.2 \\
\hline P.crispum leaves & 1.6 & \pm & 0.9 & 1.1 & \pm & 0.0 & + & 1.6 & 1.0 & \pm & 0.0 & + & 1.6 \\
\hline P.aniseum seeds & 1.1 & \pm & 0.2 & 1.0 & \pm & 0.0 & + & 1.2 & 1.0 & \pm & 0.0 & + & 1.2 \\
\hline & & & & & & & Average & 3.5 & & & & Average & 1.8 \\
\hline
\end{tabular}

a Based on the two-fold rule; (+) predictive and (-) not predictive.

b Geometric mean-fold error; ( $\leq 2)$ indicate accurate prediction and (>2) is less accurate. 


\section{Table 4}

\begin{tabular}{|c|c|c|c|c|c|c|c|c|c|c|c|c|c|}
\hline \multirow{3}{*}{ Botanical Name } & \multirow{2}{*}{\multicolumn{3}{|c|}{ Actual AUCR }} & \multicolumn{10}{|c|}{ Predicted AUCR } \\
\hline & & & & \multicolumn{5}{|c|}{ Wang et al. (2004) } & \multicolumn{5}{|c|}{ Mayhew et al. (2000) } \\
\hline & Mean & \pm & $S D$ & Mean & \pm & SD & Predictive a & GMFE b & Mean & \pm & SD & Predictive a & GMFE $b$ \\
\hline A.majus seeds & 4.7 & \pm & 1.0 & 19.8 & \pm & 0.1 & + & 4.3 & 16.6 & \pm & 1.4 & + & 3.6 \\
\hline A.archangelica roots & 2.3 & \pm & 0.6 & 3.9 & \pm & 1.6 & + & 1.6 & 1.4 & \pm & 0.3 & - & 1.6 \\
\hline C.monnieri fruits & 2.6 & \pm & 1.2 & 8.6 & \pm & 1.2 & + & 3.6 & 1.4 & \pm & 0.1 & - & 1.7 \\
\hline R.graveolens leaves & 1.7 & \pm & 0.5 & 1.7 & \pm & 0.2 & + & 1.3 & 1.6 & \pm & 0.2 & + & 1.3 \\
\hline A.pubescens roots & 1.9 & \pm & 0.8 & 1.0 & \pm & 0.0 & + & 1.8 & 1.0 & \pm & 0.0 & + & 1.8 \\
\hline A.graveolens seeds & 1.3 & \pm & 0.7 & 2.3 & \pm & 0.2 & - & 1.9 & 1.0 & \pm & 0.0 & + & 1.4 \\
\hline A.graveolens leaves & 1.2 & \pm & 0.3 & 1.3 & \pm & 0.0 & + & 1.2 & 1.0 & \pm & 0.0 & + & 1.2 \\
\hline P.crispum leaves & 1.6 & \pm & 0.9 & 1.0 & \pm & 0.0 & + & 1.6 & 1.0 & \pm & 0.0 & + & 1.6 \\
\hline P.aniseum seeds & 1.1 & \pm & 0.2 & 1.0 & \pm & 0.0 & + & 1.2 & 1.0 & \pm & 0.0 & + & 1.2 \\
\hline & & & & & & & Average & 2.0 & & & & Average & 1.7 \\
\hline
\end{tabular}

a Based on the two-fold rule; (+) predictive and (-) not predictive.

b Geometric mean-fold error; ( $\leq 2)$ indicate accurate prediction and (>2) is less accurate. 


\section{Table 5}

\begin{tabular}{|c|c|c|c|c|c|c|c|c|c|c|c|c|c|}
\hline \multirow{3}{*}{ Botanical Name } & \multirow{2}{*}{\multicolumn{3}{|c|}{ Actual AUCR }} & \multicolumn{10}{|c|}{ Predicted AUCR } \\
\hline & & & & \multicolumn{5}{|c|}{ Wang et al. (2004) } & \multicolumn{5}{|c|}{ Mayhew et al. (2000) } \\
\hline & Mean & \pm & SD & Mean & \pm & SD & Predictive a & GMFE b & Mean & \pm & SD & Predictive a & GMFE b \\
\hline A.majus seeds & 4.7 & \pm & 1.0 & 19.3 & \pm & 0.3 & + & 4.1 & 11.1 & \pm & 2.9 & + & 2.3 \\
\hline A.archangelica roots & 2.3 & \pm & 0.6 & 1.7 & \pm & 0.5 & - & 1.4 & 1.1 & \pm & 0.1 & - & 2.0 \\
\hline C.monnieri fruits & 2.6 & \pm & 1.2 & 3.3 & \pm & 0.6 & + & 1.6 & 1.1 & \pm & 0.0 & - & 2.2 \\
\hline R.graveolens leaves & 1.7 & \pm & 0.5 & 1.2 & \pm & 0.0 & + & 1.4 & 1.2 & \pm & 0.1 & + & 1.4 \\
\hline A.pubescens roots & 1.9 & \pm & 0.8 & 1.0 & \pm & 0.0 & + & 1.8 & 1.0 & \pm & 0.0 & + & 1.8 \\
\hline A.graveolens seeds & 1.3 & \pm & 0.7 & 1.3 & \pm & 0.0 & + & 1.4 & 1.0 & \pm & 0.0 & + & 1.4 \\
\hline A.graveolens leaves & 1.2 & \pm & 0.3 & 1.1 & \pm & 0.0 & + & 1.2 & 1.0 & \pm & 0.0 & + & 1.2 \\
\hline P.crispum leaves & 1.6 & \pm & 0.9 & 1.1 & \pm & 0.1 & + & 1.5 & 1.0 & \pm & 0.0 & + & 1.6 \\
\hline P.aniseum seeds & 1.1 & \pm & 0.2 & 1.0 & \pm & 0.0 & + & 1.2 & 1.0 & \pm & 0.0 & + & 1.2 \\
\hline & & & & & & & Average & 1.7 & & & & Average & 1.7 \\
\hline
\end{tabular}

a Based on the two-fold rule; (+) predictive and (-) not predictive.

b Geometric mean-fold error; ( $\leq 2)$ indicate accurate prediction and (>2) is less accurate. 
Figures

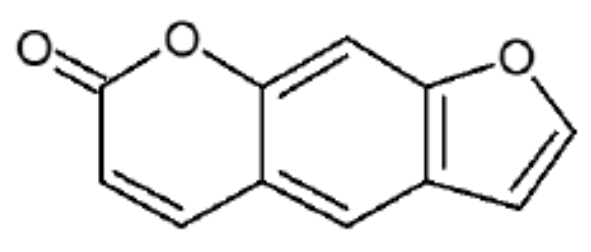

Psoralen<smiles>COc1c2ccoc2c(OC)c2oc(=O)ccc12</smiles>

Isopimpinellin (ISOP)<smiles>COc1c2occc2cc2ccc(=O)oc12</smiles>

8-methoxypsoralen (8-MOP)

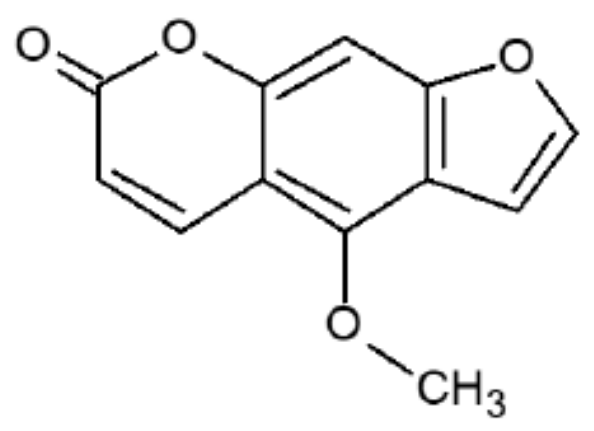

5-methoxypsoralen (5-MOP)

Figure 1

Psoralen and derivatives in this study. 


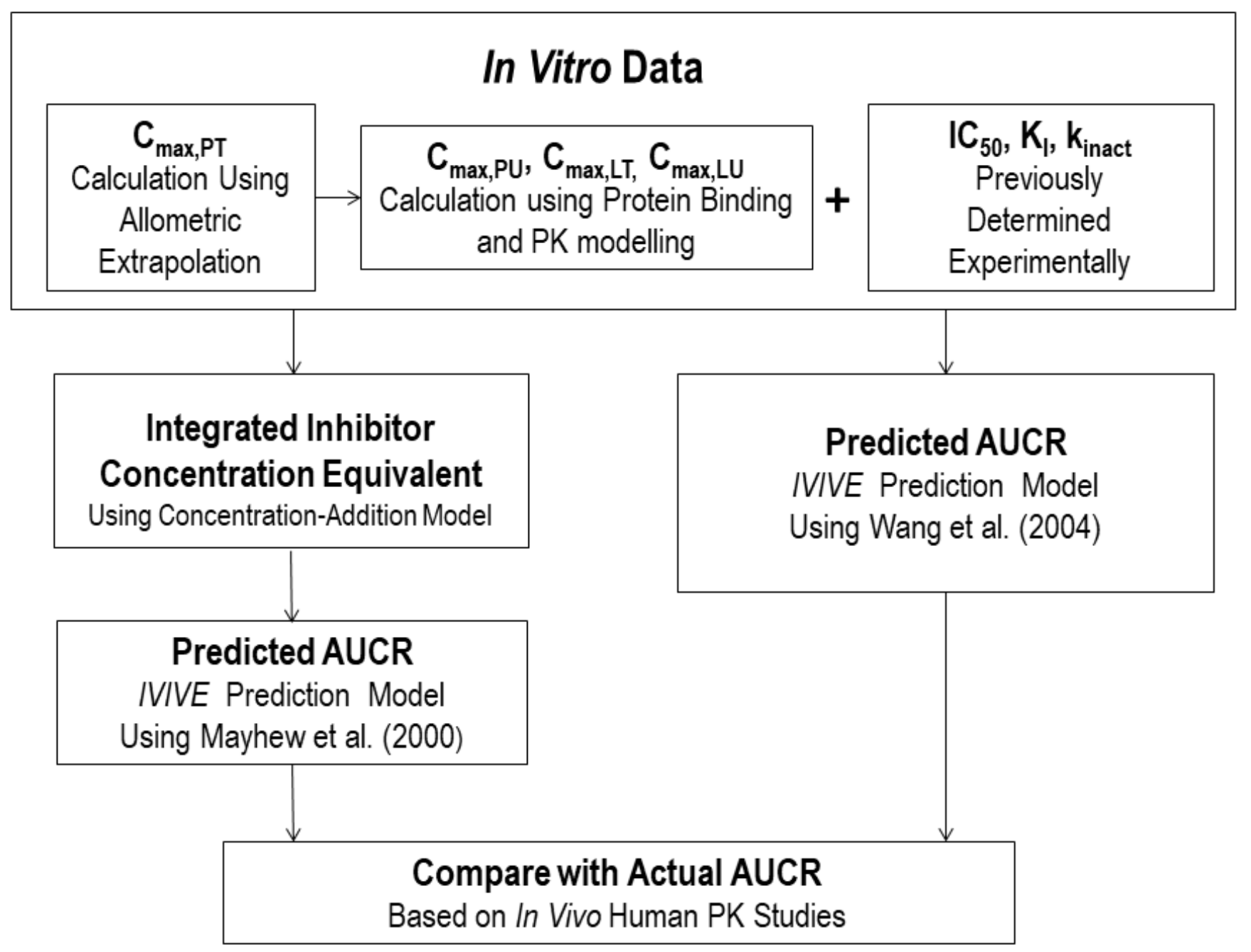

Figure 2

Flow-chart summary of experimental procedures and validation steps in the use of drug-drug interaction models to predict herb-drug interaction. 

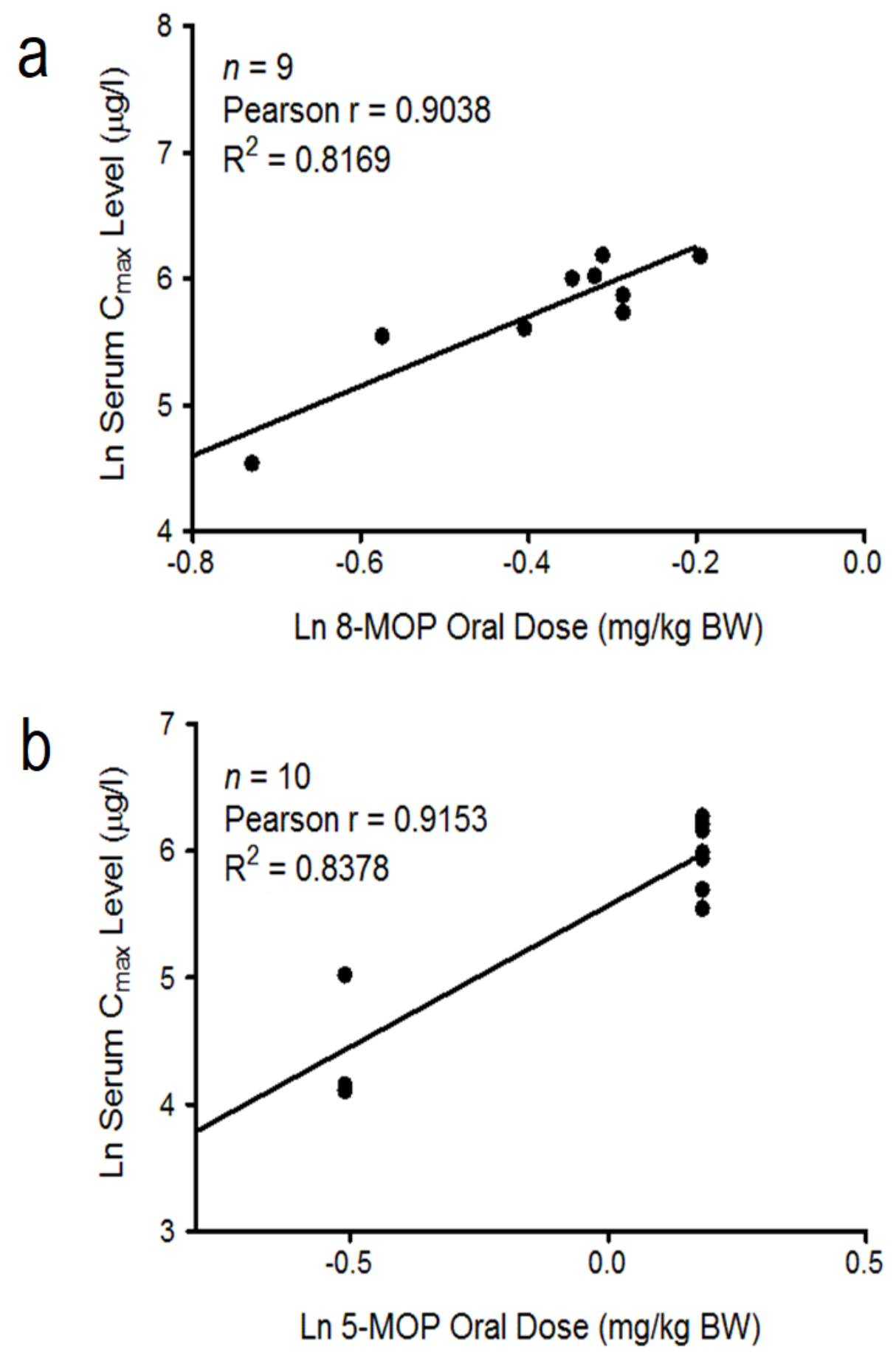

Figure 3

Ln-Ln plot of (a) 8-MOP dose versus Cmax,PT data from Schfifer-Korting and Korting (1982) and (b) LnLn plot of 5-MOP dose versus Cmax,PT data from Stolk et al. (1981). 

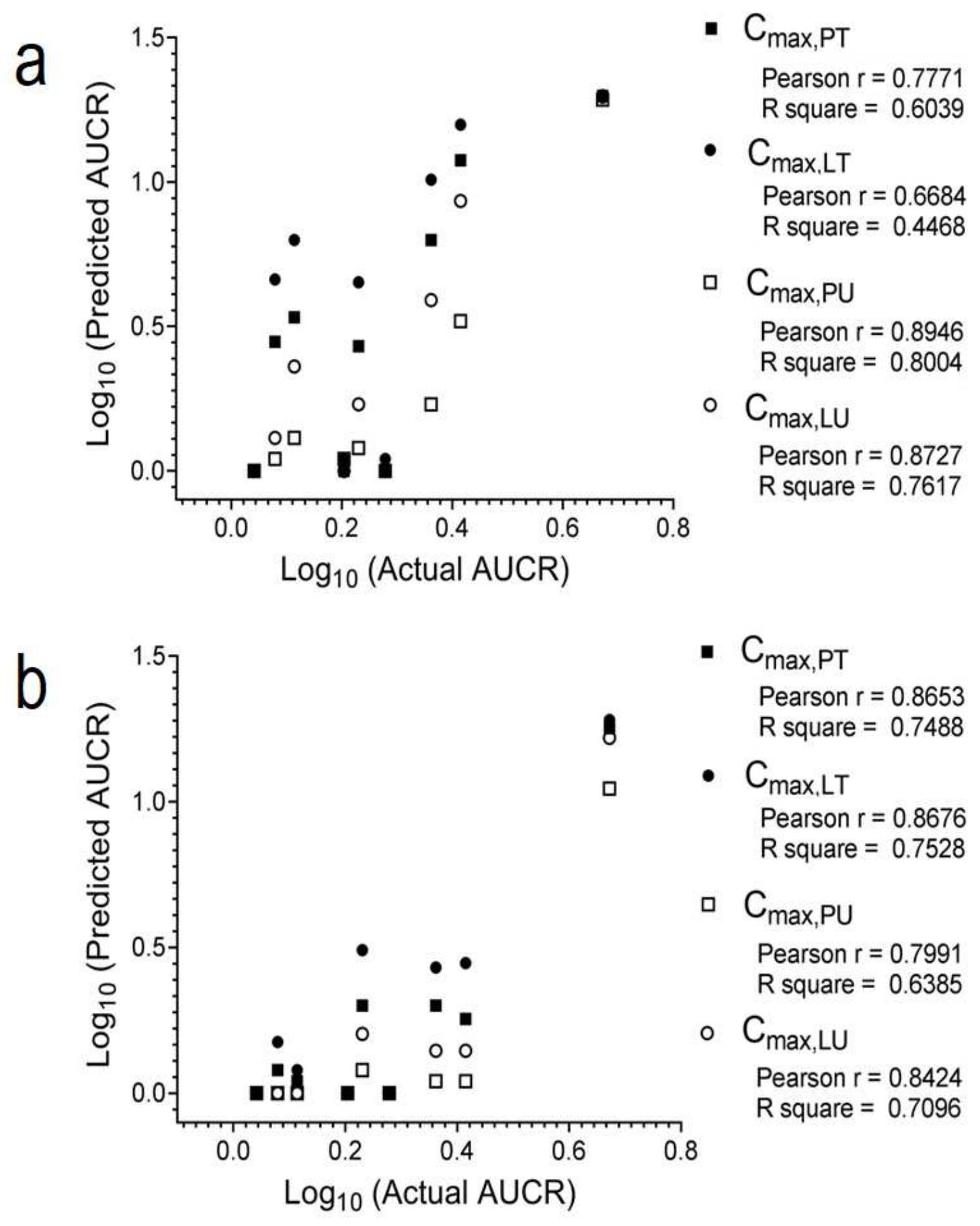

Figure 4

Correlation of (a) Wang et al. (2004) model-predicted AUCR and (b) of Mayhew et al. (2000) modelpredicted AUCR against actual AUCR using different [l]H surrogate values. 